\title{
EFICIÊNCIA DO CIPIONATO DE ESTRADIOL E DO BENZOATO DE ESTRADIOL EM PROTOCOLOS DE INDUÇÃO DA OVULAÇÃO SOBRE A DINÂMICA OVARIANA E TAXA DE CONCEPÇÃO DE FÊMEAS NELORE INSEMINADAS EM DIFERENTES MOMENTOS
}

\author{
Bruno Henrique de Araújo Andrade 1 , Priscila Assis Ferraz ${ }^{1}$, Alexandra Soares \\ Rodrigues ${ }^{1}$, Marcus Vinícius Galvão Loiola ${ }^{1}$, Marcos Chalhoub ${ }^{1}$, Antonio de Lisboa \\ Ribeiro Filho ${ }^{1}$ \\ 1 UFBA \\ Correspondência: Bruno Andrade: bruno.henryque@gmail.com
}

RESUMO: Objetivou-se avaliar a dinâmica ovariana e a taxa de concepção de fêmeas Nelore submetidas à IATF utilizando o benzoato ou o cipionato de estradiol como indutores da ovulação e inseminadas nos turnos da manhã ou da tarde. Para tanto, 296 animais receberam um dispositivo intravaginal de progesterona e a aplicação de 2,0 mg benzoato de estradiol em um dia aleatório, denominado D0. No D8 houve a remoção dos implantes, a administração de $500 \mu \mathrm{g}$ de Cloprostenol e $300 \mathrm{UI}$ de gonodotrofina coriônica equina. Neste momento, metade dos animais recebeu $1,0 \mathrm{mg}$ de cipionato de estradiol, e a outra metade $1,0 \mathrm{mg}$ de benzoato de estradiol no dia posterior (D9), constituindo os tratamentos CE e BE, respectivamente. No experimento 1,38 animais (19 de cada tratamento) tiveram a dinâmica ovariana acompanhada por ultrassonografia. No experimento 2, 258 animais distribuídos nos tratamentos (CE e BE) foram estratificados para que as inseminações ocorressem pelo turno da manhã (M) ou da tarde (T) do D10, constituindo os grupos CE-M $(n=65)$, BE-M $(n=65)$, CE-T $(n=63)$ e BE-T $(n=65)$. Não foi encontrada diferença entre os tratamentos para as variáveis: diâmetro do folículo ovulatório, momento das ovulações, taxa de ovulação e diâmetro do corpo lúteo, entretanto, os animais do grupo CE apresentaram maior taxa de crescimento folicular (CE: 0,48 mm/dia; BE: 0,29 mm/dia). Não houve diferença na taxa de concepção entre os grupos CE-M (52,3\%), BE-M (41,5\%), CE-T (41,3\%) e BE-T (49,2\%). O emprego do cipionato em substituição ao benzoato proporciona uma redução no número de manejos sem comprometer a dinâmica ovariana e os índices de concepção. Adicionalmente, a extensão no intervalo para realização das inseminações revela-se como uma alternativa aos modelos de gestão no uso desta biotecnologia.

Palavras-chave: corpo lúteo; dinâmica folicular; IATF; ultrassonografia

\section{EFFICIENCY OF ESTRADIOL CYPIONATE AND ESTRADIOL BENZOATE IN OVULATION INDUCER PROTOCOLS ON OVARIAN DYNAMICS AND CONCEPTION RATE OF NELORE FEMALES INSEMINATED AT DIFFERENT TIMES}

\begin{abstract}
We aim to evaluate the ovarian dynamics and conception rates of Nelore females subjected to TAI using benzoate or estradiol cypionate as ovulation inducers and inseminated that morning or afternoon. For this, 296 animals received an intravaginal progesterone device and 2,0 $\mathrm{mg}$ of estradiol benzoate applied on a random stage of the oestrous cycle, named D0. On D8, the devices were removed and $500 \mu \mathrm{g}$ of Cloprostenol and $300 \mathrm{IU}$ of equine chorionic gonadotropin were administered. At that time, half of the animals received $1,0 \mathrm{mg}$ of estradiol cypionate, and the other half received $1,0 \mathrm{mg}$ of estradiol benzoate on the following day (D9), establishing the EC and EB treatments respectively. On experiment 1 , 38 animals (19 for each treatment) had the ovarian dynamics accompanied by ultrasonography. On experiment 2, 258 animals were randomly distributed according to treatments (EC and EB) in order for the inseminations to occur in the morning (M) or in the afternoon (T) of D10, establishing thus groups EC-M ( $n$ $=65)$, EB-M $(n=65)$, EC-T $(n=63)$ and EB-T $(n=65)$. No difference was found between the treatments for the variables: diameter of ovulatory follicle, time of ovulation, ovulation rate and diameter of corpus luteum, however, the animals treated with estradiol cypionate presented a greater follicular growth rate (EC:0,48 mm/day; EB: 0,29 mm/day). On experiment 2, there was no difference in the conception rate between the groups EC-M (52,3\%), EB-M $(41,5 \%)$, EC-T $(41,3 \%)$ and EB-T $(49,2 \%)$. The employment of the estradiol cypionate in substitution to the estradiol benzoate provided a reduction in animal manipulation without compromising the conception rates. Additionally, the extension of the insemination interval seems to be an alternative for the management models using this technology.
\end{abstract}

Key Words: corpus luteum; follicular dynamics; FTAl; ultrasonography 


\section{INTRODUÇÃO}

A inseminação artificial (IA) é uma técnica mundialmente difundida e cumpre um importante papel no aproveitamento de indivíduos de maior mérito genético, contudo, dificuldades relacionadas com a observação do cio e momento das inseminações passam a ser um limitante em grandes rebanhos (Baruselli et al., 2004). Na pecuária de corte nacional, estes entraves são agravados pelas grandes extensões territoriais, número reduzido de funcionários em relação ao tamanho do rebanho e por particularidades do comportamento reprodutivo do gado zebu (Galina et al., 1996; Pinheiro et al., 1998).

A busca por alternativas que contornem tais empecilhos levaram ao desenvolvimento da inseminação artificial em tempo fixo (IATF), uma biotécnica aplicada para o controle das ovulações, de modo que as inseminações ocorram em horário prédeterminado. Com isso, as inseminações são programadas para um momento mais apropriado a técnicos e produtores, dispensando-se a maior limitação da IA convencional, a observação de estro (Torres-Júnior et al. 2009).

Muitos protocolos de IATF usam das propriedades da associação estrógeno/progesterona e sua capacidade em promover a regressão folicular com surgimento de uma nova onda de crescimento folicular em aproximadamente quatro dias (Bo et al., 1994; Bo et al., 1995). Os folículos recrutados da nova onda de crescimento ficam com 0 desenvolvimento modulado pelo dispositivo intravaginal de progesterona até o momento de sua remoção, quando, então, são empregados indutores hormonais, responsáveis por sincronizarem as ovulações dos folículos dominantes (Silcox et al., 1993; Baruselli et al., 2006).

$O$ benzoato de estradiol tem sido amplamente empregado como indutor da ovulação 24 h após a remoção da progesterona (Baruselli et al., 2006, Crepaldi, 2009), entretanto, pesquisas avaliaram sua substituição por um análogo hormonal, denominado cipionato de estradiol, aplicado concomitante a retirada da progesterona (Marques et al., 2004; Penteado et al., 2006; Ayres et al., 2006). Esta alteração permite eliminar o manejo adicional para aplicação do benzoato de estradiol, tornando a execução dos protocolos de sincronização mais simplificada (Crepaldi, 2009).

Esta linha de pesquisa fundamenta-se em particularidades da atividade destes fármacos sobre 0 padrão de crescimento folicular e o intervalo observado entre o tratamento e as ovulações (Souza et al., 2005). A utilização do benzoato de estradiol $24 \mathrm{~h}$ após a remoção do dispositivo de progesterona induziu o pico de LH em $16 \mathrm{~h}$, e as ovulações em 40h após a sua administração (Hanlon et al., 1997). O uso do cipionato de estradiol no momento da retirada do dispositivo de progesterona resultou em um pico de LH e ovulações, respectivamente, 38 e 66h após (Ambrose et al., 2001). Estudos conduzidos por Crepaldi et al. (2008) e Peralta-Torres et al. (2010) revelaram que a administração do cipionato de estradiol simultânea a retirada do dispositivo de progesterona promoveu a ovulação de forma sincronizada, em cerca de $72 \mathrm{~h}$ após seu uso, resultando em um momento semelhante ao protocolo em que se empregou o benzoato de estradiol $24 \mathrm{~h}$ após a remoção da progesterona.

Os resultados apresentados não são consenso na literatura. Reis et al. (2004) encontraram que o cipionato de estradiol não era eficiente em sincronizar as ovulações dos animais 
Eficiência do cipionato de estradiol e do benzoato de estradiol em protocolos de indução da ovulação sobre a dinâmica ovariana e taxa de concepção de fêmeas nelore

inseminadas em diferentes momentos

tratados com progesterona. Martins et al. (2005) acharam semelhantes taxas e tempo para as ovulações induzidas com o benzoato de estradiol e com 0 cipionato de estradiol, no entanto, classificou o cipionato de estradiol como menos eficiente em sincronizá-las.

Apesar da divergência de informação quanto à capacidade de sincronia das ovulações induzidas pelo cipionato de estradiol, a inclusão destes hormônios nos protocolos de sincronização resultaram em taxas de concepção semelhantes às obtidas com - benzoato de estradiol, nas inseminações realizadas $48 \mathrm{~h}$ (Sales et al., 2008), entre 52 e 56h (Ayres et al., 2006) e entre 54 e 58h (Penteado et al., 2006) após a remoção da progesterona.

As expectativas pela simplificação dos protocolos de sincronização levaram alguns pesquisadores a explorarem ao máximo o intervalo para realização das inseminações. Esta estratégia viabiliza um maior número de animais submetidos a um protocolo com as inseminações concentradas em um único dia. $\mathrm{O}$ conhecimento do momento das ovulações foi relatado como um evento determinante sobre os resultados das inseminações (Pursley et al., 1997; Roelofs et al. 2005; Ayres et al., 2008). Roelofs et al. (2006), verificaram melhores taxas de concepção e embriões de melhor qualidade nas inseminações realizadas entre 12 e 24h antes da ovulação. Portanto, existe um intervalo de $12 \mathrm{~h}$ para que as inseminações sejam realizadas, sem que haja comprometimento dos índices reprodutivos (Roelofs et al. 2006).

Respeitando estes intervalos para realização das inseminações, a flexibilização do momento da IA foi utilizada sem que houvesse comprometimento nos índices de concepção dos animais inseminados 48h (turno da manhã) e 54h (turno da tarde) após a remoção do dispositivo de progesterona com a utilização do cipionato de estradiol (Andrade et al., 2010) e do benzoato de estradiol (Ayres et al., 2008). Todavia, alguns pesquisadores relataram uma redução na taxa de concepção quando o cipionato de estradiol foi utilizado nos protocolos de indução da ovulação e as inseminações foram realizadas no período da tarde (Castro Júnior et al., 2008; Crepaldi, 2009).

Diante do exposto, o presente trabalho objetivou avaliar a dinâmica ovariana e a taxa de concepção de fêmeas Nelore submetidas a IATF utilizando o benzoato de estradiol ou 0 cipionato de estradiol como indutores da ovulação e inseminadas nos turnos da manhã ou da tarde.

\section{MATERIAL E MÉTODOS}

Os experimentos foram realizados durante os meses de fevereiro e março de 2011, em uma propriedade localizada no semi-árido baiano (Latitude: 13¹6'49.88"S e Longitude: $40^{\circ} 40^{\prime} 51.85^{\prime \prime}$ ), que adotava sistema extensivo de criação em pastagem de Buffel grass (Cenchrus ciliaris) com suplementação mineral e água $a d$ libitum.

Foram utilizadas 296 fêmeas Nelore com médias para idade, escore de condição corporal (escala de 1 a 5), número de partos e período pós-parto de, respectivamente: $8,22 \pm 2,47$ anos; $2,56 \pm 0,42 ; 4,12 \pm 2,02$ partos e 110,09 $\pm 30,73$ dias. Os animais foram submetidos ao exame clínicoginecológico e ultrassonografia transretal utilizando-se um transdutor linear de 6,0 MHz (Pie-Medical, Falco 100, Maastricht, Holanda) e verificou-se que $91 \%$ das fêmeas não apresentavam corpo lúteo. 


\section{Experimento 1}

Para avaliar a dinâmica folicular e o momento das ovulações, 38 animais foram uniformemente distribuídos segundo idade, escore de condição corporal, número de partos, período pós-parto e atividade ovariana (presença de corpo lúteo). Iniciou-se o tratamento em um estádio aleatório do ciclo estral, denominado dia zero (D0), quando todos os animais receberam um dispositivo intravaginal de progesterona (Sincrogest ${ }^{\circledR}$, Ouro Fino, São Paulo, Brasil) e aplicação intramuscular (i.m) de 2,0 mg de benzoato de estradiol (Gonadiolß), Schering Plough, São Paulo, Brasil). Os dispositivos foram removidos concomitante com a aplicação de $500 \mu \mathrm{g}$ de Cloprostenol i.m. (Sincrocio®), Ouro Fino, São Paulo, Brasil) e 300 UI de gonadotrofina coriônica equina (eCG) i.m. (Novormon®), Schering Plough, São Paulo, Brasil) oito dias após. Neste momento os animais foram divididos uniformemente em dois grupos experimentais, 19 animais foram tratados com $1,0 \mathrm{mg}$ de cipionato de estradiol i.m. (ECP®), Pfizer, São Paulo, Brasil), enquanto a outra metade $(\mathrm{n}=$ 19), recebeu $1,0 \mathrm{mg}$ de benzoato de estradiol i.m. pela manhã do dia posterior (D9). Todas as intervenções foram iniciadas no mesmo horário, às 8:00h (Figura 1).

No dia 10 do protocolo, $48 \mathrm{~h}$ após a remoção do dispositivo de progesterona, foi iniciada a mensuração folicular (FOL-48) utilizando imagens de ultrassom com transdutor linear de 6,0 $\mathrm{MHz} \quad$ (Pie-Medical, Falco 100, Maastricht, Holanda) a intervalo de $8 \mathrm{~h}$ entre as avaliações, até as 20:00h do dia 12 do protocolo. Outro exame ultrassonográfico foi feito no dia 20 do protocolo para confirmação da ovulação e avaliação do diâmetro do corpo lúteo (Figura 1). As mensurações foram realizadas pelo mesmo técnico. Foi considerada ovulação desaparecimento do folículo ovulatório entre dois exames ultrassonográficos consecutivos, associado à presença de um corpo lúteo no exame ultrassonográfico do dia 20 do protocolo. A taxa de crescimento folicular (TXCRE) foi obtida pela diferença entre o último diâmetro folicular antecedendo a ovulação (FOL-OV) e seu primeiro registro ao exame ultrassonográfico (FOL-48), dividido pelo tempo decorrido entre as duas observações. Os resultados da variável TX-CRE foram expressadas na unidade de milímetros por dia $(\mathrm{mm} / \mathrm{d})$.

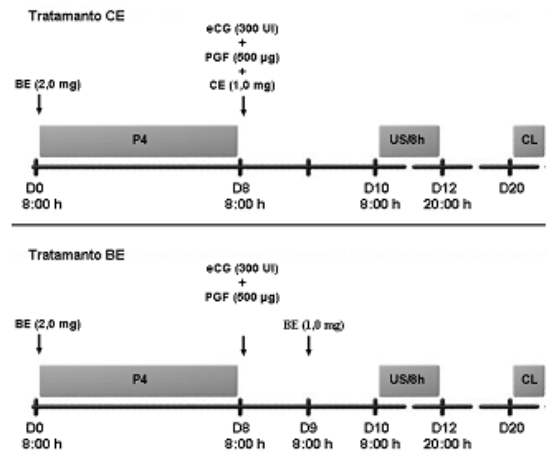

Figura 1 - Repres entação es quemática dos protocobs de sincronizaçăo da ovulaçăo e da dinâmica ovarians. Tratamento $C E-1 \mathrm{mg}$ de copionato de estradid applicado concomitante $\mathrm{s}$ remocaso da progesterona (D8). Tratamento BE- aplicaçáa de $1 \mathrm{mg}$ de benzosto de uttrassonogr afia transretal (US) a partir do D 10 , realizado a cada $8 \mathrm{~h} \mathrm{até} a$ s $20: 00 \mathrm{~h}$ do

Os dados foram processados pelo Statistical Package for Social Science (SPSS, versão 19). As diferenças nas médias entre os grupos das variáveis: a) diâmetro folicular à primeira avaliação (FOL-48), b), último diâmetro registrado antecedendo a ovulação (FOL-OV) e, c) tempo entre a retirada do dispositivo de progesterona até a ovulação (OV) foram avaliadas por meio do teste T-student. As diferenças entre as percentagens de animais que responderam ao tratamento de indução da ovulação (TXOV) foram comparadas empregando um estudo de dispersão de frequências pelo teste de Qui-quadrado ( $\chi 2)$. As diferenças nas médias entre os grupos para a variável diâmetro do corpo lúteo (CL) foram avaliadas por meio do teste T-student. Como a variável taxa de crescimento folicular (TXCRE) não apresentou distribuição normal, os grupos foram 
Eficiência do cipionato de estradiol e do benzoato de estradiol em protocolos de indução da ovulação sobre a dinâmica ovariana e taxa de concepção de fêmeas nelore

inseminadas em diferentes momentos

comparados utilizando o teste WilcoxonSigned-Ranks. Para verificar a correlação entre as variáveis FOL-OV e OV, e das variáveis FOL-OV e CL empregou-se 0 coeficiente de correlação de Pearson. Os testes foram realizados considerando um nível de significância de 0,05.

\section{Experimento 2}

Para avaliação da taxa de concepção, 258 animais uniformemente distribuídos em dois tratamentos experimentais (BE: $n=130$ e CE: $n=$ 128) foram submetidos aos mesmos protocolos hormonais descritos no experimento 1. As inseminações artificiais foram realizadas por um único técnico no D10 do protocolo. Neste momento, metade dos animais tratados com cipionato de estradiol e metade dos animais tratados com benzoato de estradiol foram separados ao acaso para que as inseminações ocorressem pelo turno da manhã (M), das 8:00h às $12: 00 \mathrm{~h}$, ou da tarde $(T)$, das $14: 00 \mathrm{~h}$ às 18:00h, constituindo assim os grupos CE-M, administração de cipionato de estradiol no D8 e inseminação pela manhã do D10 ( $n=65)$, BE-M, administração de benzoato de estradiol no D9 e inseminação pela manhã do D10 $(n=65)$, CE-T, administração de cipionato de estradiol no D8 e inseminação pela tarde do D10 $(n=63)$ e BE-T, administração de benzoato de estradiol no D9 e inseminação pela tarde do D10 $(\mathrm{n}=65) \quad$ (Figura 2). Para as inseminações empregou-se sêmen criopreservado comercializado de um único touro da raça Nelore. Os parâmetros espermáticos dessas doses de sêmen se enquadraram aos valores mínimos estabelecidos pelo Colégio Brasileiro de Reprodução Animal (Henry e Neves, 1998). No momento da IA, o sêmen foi descongelado a $37^{\circ} \mathrm{C}$ por 30 segundos.

O diagnóstico de gestação foi realizado por ultrassonografia transretal,
55 dias após as inseminações, utilizando transdutor linear de $6,0 \mathrm{MHz}$ (Pie-Medical, Falco 100, Maastricht, Holanda). Foi considerado diagnóstico de gestação positivo a presença de um feto visível com viabilidade confirmada (batimento cardíaco). A taxa de concepção foi calculada dividindo o total de vacas gestantes pelo total de vacas inseminadas.

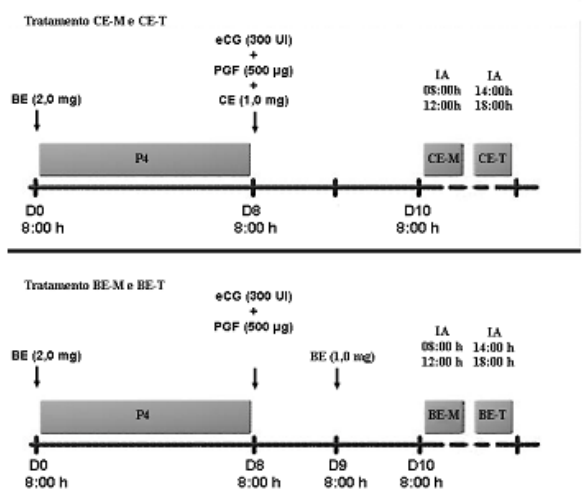

Figura 2 - Repres entaçăo esquemática dos protocolos de sincronizaçăo de ovulação e do momento das inseminaçōes. Tratamento CE-M: aplicação de $1,0 \mathrm{mg}$ de cipionato

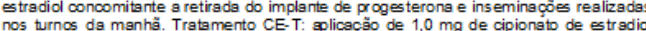
concomitante a refir ada do implante de progester on $\mathrm{e}$ ins eminacöes realizadas no turno da tarde Tratamento BE-M: aplicaçăo de $1.0 \mathrm{mg}$ de benzoato de estradiol 24 har as após a remoçẵo do implante de progesterona (D9) e inseminaçoes realizadas no turno $d$ memacäo do implante de progesterona (D9) e inseminacóes real lizadas no turno da tarde.

O experimento foi realizado atendendo os padrões de amostragem e graus de liberdade estabelecidos por Sampaio (2002). A taxa de concepção entre os grupos CE-M, BE-M, CE-T e BE-T foram comparadas empregando um estudo de dispersão de frequências pelo teste de Qui-quadrado $(\chi 2)$, disponível no pacote estatístico SPSS (versão 19), considerando nível de significância de 0,05.

\section{RESULTADOS E DISCUSSÃO}

\section{Experimento 1 \\ Diâmetro folicular}

Dois animais do Grupo BE não responderam ao tratamento de sincronização da ovulação, pois não foi verificada presença de folículo ovulatório e corpo lúteo nestes animais, respectivamente, dois dias e 12 dias após a retirada do dispositivo de progesterona. Sendo assim, os valores do diâmetro folicular, momento da 
ovulação e diâmetro do corpo lúteo destes animais não foram considerados para as análises estatísticas.

O diâmetro médio dos folículos ao início das observações, 48h após a retirada da progesterona, não diferiu entre os tratamentos (Tabela 1). Foi observado que os folículos continuaram seu crescimento até $56 \mathrm{~h}$, estabilizandose deste momento até as $64 \mathrm{~h}$, quando apresentavam média de 12,76 $\pm 1,86$ mm (CE: 13,03 $\pm 2,24$ mm e BE: 12,47 \pm 1,34 mm) (Figura 3). Resultados semelhantes foram descritos por Peralta-Torres et al. (2010), ao encontrarem que o diâmetro máximo dos folículos foram atingidos $48 \mathrm{~h}$ após a remoção da progesterona, estabilizando em cerca de 11 a $12 \mathrm{~mm}$ até o momento das ovulações.

Tabela 1 - Média e desvio padrão (SD) das variáveis: FOL-48, FOL-OV, OV, TXOV, CL e TXCRE $(n=38)$.

\begin{tabular}{|c|c|c|c|}
\hline & $\begin{array}{c}\mathrm{CE}^{7} \\
\text { Média } \pm(\mathrm{SD})\end{array}$ & $\begin{array}{c}\mathrm{BE}^{8} \\
\text { Média } \pm(\mathrm{SD})\end{array}$ & $\begin{array}{c}\text { Valor de } \\
\text { P }\end{array}$ \\
\hline $\mathrm{FOL}^{-48^{1}(\mathrm{~mm})}$ & $11,86 \pm 2,57$ & $11,84 \pm 1,59$ & 0,977 \\
\hline FOL-OV ${ }^{2}(\mathrm{~mm})$ & $13,31 \pm 2,25$ & $12,69 \pm 1,21$ & 0,303 \\
\hline $\mathrm{OV}^{5}(\mathrm{~h})$ & $73,74 \pm 5,75$ & $74,76 \pm 7,30$ & 0,642 \\
\hline $\operatorname{TXOV}^{4}(\%)$ & 100,00 & 89,50 & 0,146 \\
\hline $\mathrm{CL}^{5}(\mathrm{~mm})$ & $16,96 \pm 3,74$ & $17,42 \pm 2,98$ & 0,689 \\
\hline $\operatorname{TXCRE}^{\circ}(\mathrm{mm} / \mathrm{d})$ & $0,48 \pm 0,49^{\circ}$ & $0,29 \pm 0,28^{\mathrm{a}}$ & 0,000 \\
\hline \multicolumn{4}{|c|}{ 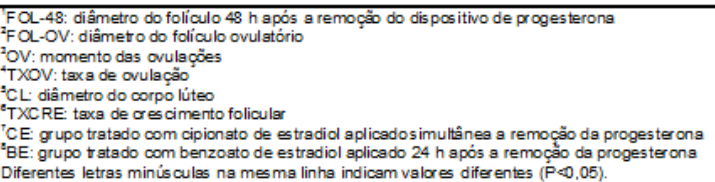 } \\
\hline
\end{tabular}

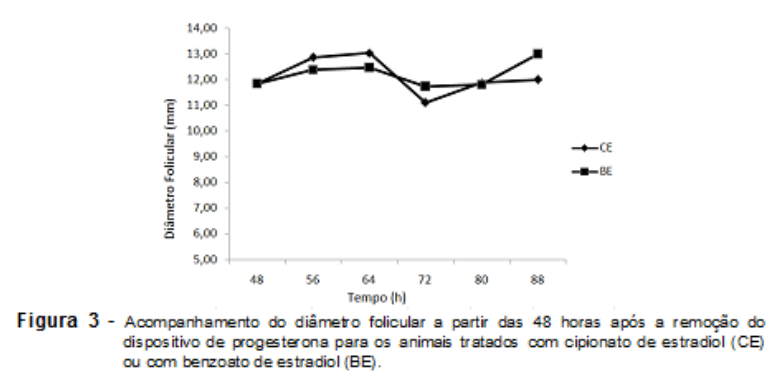

A utilização de estratégias buscando aumentar o diâmetro do folículo ovulatório são alternativas para melhorar a eficiência dos protocolos de sincronização. Nos trabalhos de Vasconcelos et al. (2001) e Ferraz et al. (2010), os autores relacionaram folículos com maiores diâmetros ovulatórios a índices de concepção superiores. Sendo assim, a utilização de indutores que resultem em folículos de maior tamanho poderiam incrementar os resultados dos protocolos de sincronização.

$\mathrm{Na}$ comparação entre os tratamentos $\mathrm{CE}$ e $\mathrm{BE}$ foi verificado que o emprego do cipionato de estradiol e do benzoato de estradiol resultaram em folículos com diâmetros ovulatórios semelhantes (Tabela 1). Crepaldi (2009) também relatou não haver diferenças sobre o diâmetro médio dos folículos ovulatórios para os animais que receberam cipionato de estradiol no dia $8(14,0 \pm 0,6 \mathrm{~mm})$, e o benzoato de estradiol $(13,6 \pm 0,5 \mathrm{~mm})$ no dia 9 do protocolo. Da mesma forma, Reis et al. (2004), ao trabalharem com aplicações do benzoato de estradiol e do cipionato de estradiol em ambos os dias, 8 e 9 do protocolo, observaram um menor diâmetro folicular apenas nos animais cuja administração do benzoato de estradiol ocorreu simultânea a remoção do progestágeno (dia 8).

\section{Ovulações}

$O$ intervalo entre a retirada do dispositivo de progesterona $e$ as ovulações foi similar entre os dois tratamentos (Tabela 1). Os resultados assemelham-se aos obtidos por Crepaldi et al. (2008) (cipionato de estradiol: $72,0 \pm 2,0 \mathrm{~h}$, benzoato de estradiol: $72,0 \pm 0,0 \mathrm{~h})$, e Sales et al. (2008) (cipionato de estradiol: 74,5h, benzoato de estradiol: $76,1 \mathrm{~h}$ ), os quais observaram não haver diferença entre a utilização destes indutores e o momento em que as ovulações ocorriam. PeraltaTorres et al. (2010) sugeriram que ambos indutores foram igualmente eficientes em promover o aumento da frequência dos pulsos de $\mathrm{LH}$ e sincronizar as ovulações dos animais tratados com cipionato de estradiol $(70,0$ $\pm 7,7 \mathrm{~h})$ e com o benzoato de estradiol (73,0 $\pm 6,5 \mathrm{~h})$, sobretudo, foi evidente 
Eficiência do cipionato de estradiol e do benzoato de estradiol em protocolos de indução da ovulação sobre a dinâmica ovariana e taxa de concepção de fêmeas nelore

inseminadas em diferentes momentos

uma tendência na antecipação deste evento em cerca de $15 \mathrm{~h}$, em relação ao grupo controle, no qual não empregaram algum indutor $(88,0 \pm$ 22,3h).

Todavia, alguns estudos apontaram que a utilização do cipionato de estradiol resultava em atraso nas ovulações, as quais surgiam de forma não sincronizada (Reis et al., 2004) ou menos sincronizada (Martins et al., 2005) do que o obtido com a utilização do benzoato de estradiol. Paradoxalmente, quando Crepaldi (2009) utilizou dispositivos de progesterona reutilizados, propôs que a menor disponibilidade de progesterona nestes dispositivos estaria favorecendo a antecipação das ovulações, prejudicando desta forma, os índices de concepção apenas nos animais em que foi empregado o cipionato de estradiol. Entretanto, no presente experimento este mecanismo não foi observado, podendo ser justificado por ter sido utilizado apenas dispositivos novos de progesterona nos protocolos.

\section{Taxa de Ovulação}

A taxa de ovulação (TXOV) não diferiu entre os tratamentos CE e BE (Tabela 1), corroborando com resultados de outros pesquisadores (Reis et al., 2004, Martins et al., 2005, Crepaldi, 2009, Peralta-Torres et al., 2010). Dois animais do tratamento BE não ovularam e um deles, embora tenha apresentado um folículo de $11,5 \mathrm{~mm}$ de diâmetro, este folículo começou a entrar em atresia a partir do dia 11 do protocolo, provavelmente, por não ter alcançado capacidade ovulatória. Sartori et al. (2001), ao trabalharem com vacas holandesas (Bos taurus taurus), observaram que os folículos começavam a adquirir capacidade ovulatória a partir de $8,5 \mathrm{~mm}$ de diâmetro, ainda assim, apenas $80 \%$ dos folículos com mais de $10 \mathrm{~mm}$ foram eficientes em responder ao tratamento de indução da ovulação; da mesma forma, Gimenes et al. (2008), ao induzirem a ovulação em fêmeas Nelore, constataram que $10 \%$ dos folículos com mais de $10 \mathrm{~mm}$ não responderam a aplicação do $\mathrm{LH}$. Portanto, nem todos os folículos que ultrapassam $10 \mathrm{~mm}$ de diâmetro alcançam a capacidade ovulatória. Além disso, fatores relacionados à sensibilidade individual à progesterona liberadas pelos dispositivos, podem interferir sobre os resultados de ovulação, por exercer feedback negativo ao aumento da frequência dos pulsos de LH (Roberson et al., 1989; Carvalho et al., 2008; Cipriano et al., 2011). O outro animal que não respondeu ao tratamento de indução da ovulação apresentou folículo de $6 \mathrm{~mm}$ de diâmetro, permanecendo estático durante as observações. A ausência de crescimento observada neste folículo pode ser explicada pelo fato destas estruturas apresentarem uma escassez de receptores de LH como apontado por Nogueira et al. (2005) e Gimenes et al. (2008).

A distribuição das ovulações mostrou-se bastante uniforme entre os animais que receberam cipionato de estradiol e benzoato de estradiol: apenas 7,14\% (1/19) dos animais de cada tratamento ovularam no intervalo até $64 \mathrm{~h}, 7,14 \%(\mathrm{n}=1 / 19)$ ovularam após as $80 \mathrm{~h}$ e $31,58 \%$ (6/19) no intervalo 72-80h. A única diferença numérica verificada ocorreu no intervalo $64-72 \mathrm{~h}$, quando $57,89 \%$ (11/19) dos animais que receberam cipionato de estradiol ovularam, contra 47,37\% (9/19) dos animais que receberam benzoato de estradiol.

\section{Corpo lúteo}

Os diâmetros dos corpos lúteos não diferiram entre os tratamentos (Tabela 1). Segundo Viana et al. (1999) o diâmetro máximo do corpo lúteo é alcançado entre os dias 7 e 16 do ciclo 
(9,64 $\pm 0,25$ dias). O diâmetro médio dos corpos lúteos apresentado por estes autores em vacas Gir, comparam-se ao deste experimento e aos encontrados por Figueiredo et al. (1997) em vacas Nelore (15,90 a 17,69mm). Baruselli et al. (2003) e Vasconcelos et al. (2001) verificaram que corpos lúteos com maior área determinaram maiores taxas de concepção, atribuindo tal relação à sua maior capacidade de produção de progesterona. Neste aspecto, ambos indutores resultaram em corpos lúteos de diâmetro similar, corroborando com os achados de Reis et al. (2004), os quais verificaram não haver diferença entre as áreas dos corpos lúteos com a utilização do cipionato de estradiol $(3,23$ $\left.\pm 0,24 \mathrm{~cm}^{2}\right)$ aplicado concomitante a remoção da progesterona, ou da aplicação do benzoato de estradiol $(3,04$ $\pm 0,27 \mathrm{~cm}^{2}$ ) realizada $24 \mathrm{~h}$ depois.

\section{Taxa de Crescimento Folicular}

As taxas de crescimento observadas para ambos os tratamentos (Tabela 1) foram inferiores à média reportada anteriormente para Bos taurus indicus, de 0,9mm/dia (Castilho et al., 2007; Gimenes et al., 2008). Esta diferença pode ter ocorrido pelo fato dos valores iniciais do diâmetro folicular terem sido aferidos $48 \mathrm{~h}$ após a remoção da fonte de progesterona, desconsiderando assim, o crescimento que houve desde 0 início de desenvolvimento da onda.

A taxa de crescimento do folículo ovulatório dos animais tratados com cipionato de estradiol foi significativamente superior àquela observada para os animais do tratamento BE (Tabela 1), demonstrando que os folículos das fêmeas tratadas com cipionato de estradiol, como indutor da ovulação, apresentaram uma maior habilidade de crescimento.

Considerando 0 estradiol-17 $\beta$ como a molécula cuja função biológica esta envolvida na habilidade dos folículos em adquirirem receptores de $\mathrm{LH}$ e no crescimento folicular (Stewart et al., 1995), alguns estudos buscaram definir diferentes perfis em sua disponibilidade após o emprego dos ésteres, cipionato de estradiol e benzoato de estradiol. Souza et al. (2005) caracterizaram a circulação do estradiol-17 $\beta$ de animais submetidos à aplicação de 1,0mg destes fármacos e observaram que as concentrações de estradiol-17 $\beta$ tendiam a aumentar $24 \mathrm{~h}$ após a administração do cipionato de estradiol, e um aumento significativo foi verificado $4 \mathrm{~h}$ após a administração do benzoato de estradiol. Estes resultados indicam que o emprego do cipionato de estradiol e do benzoato de estradiol, quando utilizados em momentos distintos do protocolo (D8 e D9, respectivamente), deve resultar em folículos submetidos a maiores quantidades de estradiol-17 $\beta$ em um momento comum - cerca de $28 \mathrm{~h}$ após a remoção da progesterona.

$$
\text { Vynckier et al. }
$$
administraram $10,0 \mathrm{mg}$ de cipionato e 2,0mg de benzoato de estradiol e chegaram as seguintes observações: o cipionato de estradiol disponibilizava um menor pico de estradiol-17 $\beta$ (cipionato de estradiol: $56-128 \mathrm{pg} / \mathrm{mL}$ e benzoato de estradiol: $222-320 \mathrm{pg} / \mathrm{mL}$ ), que surgia mais tardiamente (cipionato de estradiol: 13-31h e benzoato de estradiol: 1-23h), mas que persistia por um maior intervalo até retornarem aos níveis basais. Burke et al. (2000) utilizaram 1,0mg do benzoato de estradiol e observaram concentrações elevadas do estradiol$17 \beta 4 \mathrm{~h}$ após o tratamento, os quais mantinham-se elevadas $(12,0 \mathrm{pg} / \mathrm{mL})$ por um período de 24h. Perry e Perry (2008) e Amer (2008), por sua vez, ao trabalharem com a mesma dosagem utilizando o cipionato de estradiol, observaram que as maiores concentrações do estradiol-17 $\beta$ eram atingidas $24 \mathrm{~h}$ após o tratamento. Considerando estas diferenças, é 
Eficiência do cipionato de estradiol e do benzoato de estradiol em protocolos de indução da ovulação sobre a dinâmica ovariana e taxa de concepção de fêmeas nelore

inseminadas em diferentes momentos

possível que níveis de estradiol-17 $\beta$ passem a agir mais precocemente sobre a dinâmica folicular dos animais tratados com cipionato de estradiol, principalmente, níveis que antecedendo seu pico de concentração, estejam a contribuir para uma maior taxa de crescimento destes folículos.

\section{Correlações}

A partir das $64 \mathrm{~h}$, o diâmetro médio dos folículos foi representado graficamente por uma tênue diminuição para os animais do tratamento $\mathrm{CE}$ (Figura 3). Esta observação pode estar relacionada ao achado de Gimenes et al. (2008), de que quanto maior o tamanho do folículo, menor é o tempo para que estes folículos ovulem. Tal consideração foi confirmada para os animais do tratamento $\mathrm{CE}$, onde verificou-se uma correlação moderada e negativa entre o diâmetro do folículo ovulatório e o momento da ovulação ( $R$ $=-0,480 ; P=0,038)$. No entanto, esta associação não ocorreu para os animais tratados com benzoato de estradiol ( $R=$ $-0,204 ; P=0,431)$.

O diâmetro do folículo ovulatório mostrou-se positiva e moderadamente correlacionada com o diâmetro do CL ( $R$ $=0,416 ; P=0,012)$. Relacionado $a$ estas variáveis, Baruselli et al. (2003) verificaram que corpos lúteos com maior área determinaram maiores concentrações de progesterona e taxas de concepção. Vasconcelos et al. (2001) observaram que folículos menores estavam relacionados a corpos lúteos de menor diâmetro e a baixa fertilidade, atribuindo estes achados à menor capacidade dos corpos lúteos em produzir progesterona. Esta argumentação é controversa aos achados de Robinson et al. (2005), os quais não confirmaram tal correlação, contudo, folículos maiores estiveram relacionados a uma maior produção de estrógenos no período pré-ovulatório e a expressão mais precoce de progesterona. Estes autores concluíram que o desenvolvimento embrionário e sua manutenção durante os estágios iniciais da gestação estão mais relacionados ao diâmetro do folículo ovulatório, do que propriamente ao diâmetro do corpo lúteo.

\section{Experimento 2}

A taxa de concepção média foi de $46,1 \%$ e não houve diferença entre os grupos experimentais CE-M, BE-M, CE$\mathrm{T}$ e BE-T (Tabela 2). Meneghetti et al. (2009) também não verificaram diferenças na taxa de concepção entre os animais que receberam cipionato de estradiol concomitante a remoção do dispositivo de progesterona ou $\mathrm{o}$ benzoato de estradiol aplicado $24 \mathrm{~h}$ depois, quando as inseminações foram realizadas no turno da manhã (cipionato de estradiol: $56,7 \%$ e benzoato de estradiol: 57,1\%). Da mesma forma, Ayres et al. (2006) e Penteado et al. (2006) não encontraram diferenças entre os animais tratados com estes indutores e inseminados, respectivamente, nos intervalos 52 - 56h e 54 - 58h após a remoção da progesterona.

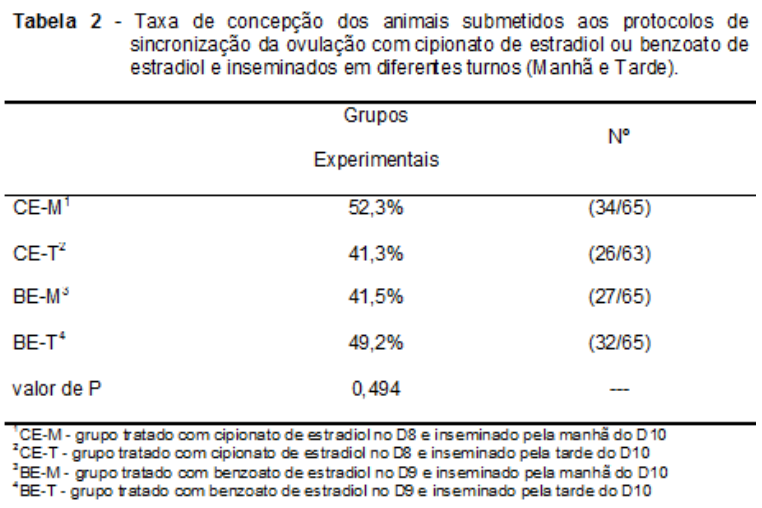

Estes resultados confirmam a possibilidade da realização das inseminações em diferentes turnos (manhã e tarde) sem que haja comprometimento nos índices de concepção, tornando possível a flexibilização do momento das inseminações e a inclusão de um maior número de animais no protocolo. Além 
disso, os resultados encontrados nesta pesquisa e os reportados por diversos pesquisadores demonstram que a utilização do cipionato de estradiol proporciona uma redução no número de manejos e aumenta a praticidade dos programas de IATF, sobretudo em grandes rebanhos (Ayres et al. 2006; Castro-Júnior et al. 2008; Crepaldi, 2009).

Todavia, Castro Júnior et al. (2008), ao trabalharem com dispositivos previamente utilizados por nove dias, observaram que animais tratados com cipionato de estradiol quando inseminados à tarde apresentavam comprometimento nos índices de prenhez (cipionato de estradiol: $38,5 \%$ e benzoato de estradiol: $60,4 \%$ ), sendo o mesmo não observado entre os animais inseminados no turno da manhã (cipionato de estradiol: 49,5\% e benzoato de estradiol: $54,4 \%$ ). Crepaldi (2009) relatou uma situação semelhante ao avaliar a taxa de concepção entre animais que receberam dispositivos novos ou previamente utilizados. Em seu estudo, animais que receberam o cipionato de estradiol e foram inseminados à tarde tiveram seus índices comprometidos apenas quando trabalhou-se com dispositivos previamente utilizados. As diferenças apresentadas por estes autores podem estar relacionadas ao número de usos do dispositivo - a menor disponibilidade de progesterona liberada por estes dispositivos estaria predispondo um aumento na pulsatilidade do LH (Carvalho et al. 2008), antecipando as ovulações. Desta forma, quando as inseminações são realizadas pela tarde, o tempo decorrido para as ovulações fica reduzido, interferindo nos resultados de concepção. Contudo este mecanismo não foi verificado nesta pesquisa, pois não houve o emprego de dispositivos de progesterona reutilizados.

\section{CONCLUSÃO}

O emprego do cipionato de estradiol ou do benzoato de estradiol como indutores da ovulação mostrou eficiência semelhante em relação à dinâmica folicular. A utilização do cipionato de estradiol em substituição ao benzoato de estradiol não comprometeu os índices de concepção dos animais inseminados $48 \mathrm{~h}$ (manhã) ou $54 \mathrm{~h}$ (tarde) após a remoção da progesterona, no entanto, sua escolha torna a execução dos protocolos mais simplificada, proporcionando uma redução no número de manejo do protocolo e consequentemente um menor custo logístico e operacional. Adicionalmente, a extensão no intervalo para realização das inseminações (manhã e tarde), revela-se como uma alternativa aos modelos de gestão no uso desta biotecnologia, permitindo a inclusão de um maior número de animais submetidos a um protocolo e inseminados no mesmo dia.

\section{AGRADECIMENTOS}

Agradecemos à Coordenação de Aperfeiçoamento de Pessoal de Nível Superior (CAPES), pelo apoio financeiro em forma de bolsa de estudo indispensável para realização deste trabalho.

\section{REFERÊNCIAS}

AMBROSE, D.J.; RAJAMAHENDRAN, R.; KASTELIC, J.P. et al. Synchronization of ovulation and conception rates in Holstein heifers given an intravaginal progesteronereleasing device (CIDR), and estradiol cypionate, porcine LH or gonadotropin releasing hormone. Archiv Tierzucht, v.44, p.77-79, 2001.

AMER, H.A. Oestrus synchronization in high lactating dairy cows. Mljekarstvo, v.58, n.1, p.33-46, 2008. 
Eficiência do cipionato de estradiol e do benzoato de estradiol em protocolos de indução da ovulação sobre a dinâmica ovariana e taxa de concepção de fêmeas nelore

ANDRADE, B. H. A.; FERRAZ, P. A.; LOIOLA, M. V. G.; et al. Effect of artificial insemination moment on conception rate in a protocol for FTAI. Acta Scienciae Veterinariae, v.38, n.2, p.359, 2010.

AYRES, H.; MARTINS, C.M.; FERREIRA, R.M. et al. Effect of timing of estradiol benzoate administration upon synchronization of ovulation in suckling Nelore cows (Bos indicus) treated with a progesterone-releasing intravaginal device. Animal Reproduction Science, v.109, n.1, p.77-87, 2008.

AYRES, H.; PENTEADO, L.; TORRES JÚNIOR, J.R.S. et al. Taxa de concepção de vacas nelore lactantes sincronizadas com implate auricular de progestágeno associado ao benzoato ou ao cipionato de estradiol. Acta Scienciae

Veterinariae, v.34, p.410, 2006.

BARUSELLI, P.S.; AYRES, H.; SOUZA, A.H. et al. Impacto da IATF na eficiência reprodutiva em bovinos de corte. In: SIMPÓSIO INTERNACIONAL DE REPRODUÇÃO ANIMAL APLICADA, II., 2006, Londrina. Anais... São Paulo: VRA-FMVZ, 2006. p.103-136.

BARUSELLI, P.S.; MARQUES, M.O.; CARVALHO, N.A.T. et al. Dinâmica folicular e taxa de prenhez em novilhas receptoras de embrião (Bos taurus indicus x Bos taurus taurus) tratadas com o protocolo "Ovsynch" para inovulação em tempo fixo. Brazilian Journal of Veterinary Research and Animal Science, v.40, suppl.2, p.96-106, 2003.

BARUSELLI, P.S.; REIS, E.L.; MARQUES, M.O. et al. The use of hormonal treatments to improve reproductive performance of anestrous beef cattle in tropical climates. Animal Reproduction Science, v.82-83, p.479-486, 2004.

BO, G.; CACCIA, M.; MARTÍNEZ, M. et al. The use of estradiol-17b and progestogen treatment for the control of follicular wave dynamics in beef cattle. Theriogenology, v.40, p.165, 1994.

BO, G.A.; ADAMS, G.P.; PIERSON, R.A. et al. Exogenous control of follicular wave emergence in cattle. Theriogenology, v.43, n.1, p.31-40, 1995.

BURKE, C.R. et al. Use of a small dose of estradiol benzoate during diestrus to synchronize development of the ovulatory follicle in cattle. Journal of Animal Science, v.78, n.1, p.145-151, 2000.

CARVALHO, J.B.; CARVALHO, N.A.; REIS, E.L. et al. Effect of early luteolysis in progesteronebased timed Al protocols in Bos indicus, Bos indicus x Bos taurus, and Bos taurus heifers. Theriogenology, v.69, n.2, p.167-75, 2008. inseminadas em diferentes momentos CASTILHO, C.; GARCIA, J.M.; RENESTO, A. et al. Follicular dynamics and plasma FSH and progesterone concentrations during follicular deviation in the first post-ovulatory wave in Nelore (Bos indicus) heifers. Animal

Reproduction Science, v.98, n.3-4, p.189-196, 2007.

CASTRO JÚNIOR, J.D.; SALES, J.N.S.; CREPALDI, G.A. et al. Efeito do tratamento com diferentes indutores da ovulação (cipionato e benzoato de estradiol) e do momento da inseminação artificial (48 e 54h após a retirada do implante de norgestomet) na taxa de prenhez de vacas nelore (Bos indicus) inseminadas em tempo fixo. Acta Scienciae Veterinariae, v.36, p.617, 2008.

CIPRIANO, R.S.; CARVALHO, B.A.; MARANGONI, N.R. et al. LH and FSH concentration and follicular development in Nellore heifers submitted to fixed-time artificial insemination protocols with different progesterone concentrations. Animal Reproduction Science, v.27, n.1, p.16-22, 2011.

CREPALDI, G. A. Eficácia de diferentes protocolos de indução da ovulação e de intervalos de inseminação em vacas de corte submetidas à IATF. 2009. São Paulo. 88f. Dissertação (Mestrado em Medicina Veterinária) - Curso de Pós-Graduação em Reprodução Animal, Universidade de São Paulo.

CREPALDI, G.A.; SALES, J.N.S.; GIROTTO, R.W. et al. Momento da ovulação e taxa de concepção de vacas nelore tratadas com cipionato ou benzoato de estradiol para induzir a ovulação em protocolos de IATF. Acta

Scienciae Veterinariae, v.36, p.464, 2008.

FERRAZ, P. A.; RODRIGUES, A. S.; LOIOLA, M. V. G.; et al. Diâmetro do folículo préovulatório no momento da inseminação artificial em tempo fixo em vacas Nelore. Acta Scienciae Veterinariae, v.38, n.2, p.81, 2010.

FIGUEIREDO, R. A.; BARROS, C. M.; PINHEIRO, O. et al. Ovarian follicular dynamics in nelore breed (Bos indicus) cattle.

Theriogenology, v.47, n.8, p.1489-1505, 1997.

GALINA, C.S.; ORIHUELA, A.; RUBIO, I.

Behavioural trends affecting oestrus detection in Zebu cattle. Animal Reproduction Science, v.42, n.1-4, p.465-470, 1996.

GIMENES, L.U.; SÁ FILHO, M.F.; CARVALHO, N.A.T. et al. Follicle deviation and ovulatory capacity in Bos indicus heifers.

Theriogenology, v.69, n.7, p.852-858, 2008. 
HANLON, D.W.; WILLIAMSON, N.B.; WICHTEL, J.J. et al. Ovulatory responses and plasma luteinizing hormone concentrations in dairy heifers after treatment with exogenous progesterone and estradiol benzoate. Theriogenology, v.47, n.5, p.963-75, 1997.

HENRY M, NEVES JP. Manual para Exame Andrológico e Avaliação de Sêmen Animal. Belo Horizonte: Colégio Brasileiro de Reprodução Animal, 1998.

MARQUES, M. O.; AYRES, H.; REIS, E. L.; et al. Efeito do cipionato e do benzoato de estradiol na taxa de prenhez de vacas nelore inseminadas em tempo fixo. Acta Scienciae Veterinariae, v.32, p.222, 2004.

MARTINS, C.M.; CASTRICINI, E.S.C.; SÁ FILHO, M.F. et al. Dinâmica folicular de vacas nelore tratadas com Cipionato ou Benzoato de estradiol em protocolos de inseminação artificial em tempo fixo. Acta Scienciae Veterinariae, v.33, p.285, 2005.

MENEGHETTI, M.; SA FILHO, O.G.; PERES, R.F. et al. Fixed-time artificial insemination with estradiol and progesterone for Bos indicus cows I: basis for development of protocols.

Theriogenology, v.72, n.2, p.179-89, 2009.

NOGUEIRA, M.F.G.; PINTO, M.G.L.; RAINHO, C. A. et al. Expressão das isoformas do gene codificador do receptor de LH em células da teca e da granulosa de folículos antrais bovinos. Acta Scienciae Veterinariae, v.33, p.337, 2005.

PENTEADO, L.; AYRES, H.; TORRES JÚNIOR, J.R.S. et al. Taxa de concepção de vacas nelore lactantes sincronizadas com implante auricular de progestágeno associado ao benzoato ou ao cipionato de estradiol. Acta Scienciae Veterinariae, v.34, p.401, 2006.

PERALTA-TORRES, J.; LOPEZ, J.A.; CASTRO, F.C. et al. Comparison of estradiol cypionate and estradiol benzoate effects on ovaric activity, estrus and ovulation on anestrus Bos indicus cows. Journal of Animal and Veterinary Advances, v.9, n.3, p.466-470, 2010.

PERRY, G.A.; PERRY, B.L. Effect of preovulatory concentrations of estradiol and initiation of standing estrus on uterine $\mathrm{pH}$ in beef cows. Domestic Animal Endocrinology, v.34, n.3, p.333-338, 2008.

PINHEIRO, O.L.; BARROS, C.M.; FIGUEIREDO, R.A. et al. Estrous behavior and the estrus-to-ovulation interval in nelore cattle (Bos indicus) with natural estrus or estrus induced with prostaglandin F2 $\alpha$ or norgestomet and estradiol valerate. Theriogenology, v.49, n.3, p.667-681, 1998.
PURSLEY, J. R.; WILTBANK, M. C.; STEVENSON, et al. Pregnancy Rates Per Artificial Insemination for Cows and Heifers Inseminated at a Synchronized Ovulation or Synchronized Estrus. Journal of dairy science, v.80, n.2, p.295-300, 1997.

REIS, E.L.; GIMENES, L.U.; MARQUES, M.O. et al. Efeitos do cipionato e do benzoato de estradiol na dinâmica folicular e luteínica de vacas Nelore. Acta Scienciae Veterinariae, v.32, p.236, 2004.

ROBERSON, M.S.; WOLFE, M.W.; STUMPF, T.T. et al. Luteinizing hormone secretion and corpus luteum function in cows receiving two levels of progesterone. Biology of

Reproduction, v.41, n.6, p.997-1003, 1989.

ROBINSON, R.S.; HAMMOND, A.J.; HUNTER, M.G. et al. The induction of a delayed postovulatory progesterone rise in dairy cows: a novel model. Domestic Animal Endocrinology, v.28, n.3, p.285-295, 2005.

ROELOFS, J.B.; GRAAT, E.A.M.; MULLAART, $E$. et al. Effects of insemination-ovulation interval on fertilization rates and embryo characteristics in dairy cattle. Theriogenology, v.66, n.9, p.2173-2181, 2006.

ROELOFS, J.B.; VAN EERDENBURG, F.J.C.M.; et al. Various behavioral signs of estrous and their relationship with time of ovulation in dairy cattle. Theriogenology, v.63, n.5, p.1366-1377, 2005.

SALES, J.N.S.; CREPALDI, G.A.; CARVALHO, J.B.P. et al. Momento da ovulação e taxa de concepção de vacas nelore tratadas com diferentes fontes de cipionato ou de benzoato de estradiol para induzir a ovulação em protocolos de IATF. Acta Scienciae Veterinariae, v.36, p.486, 2008.

SAMPAIO, I.B.M. Estatística aplicada à experimentação animal. 2. ed. Belo Horizonte: FEPMVZ, 2002.

SARTORI, R.; FRICKE, P.M.; FERREIRA, J.C.P. et al. Follicular deviation and acquisition of ovulatory capacity in bovine follicles. Biology of Reproduction, v.65, n.5, p.1403-1409, 2001.

SILCOX, R.; POWELL, K.; KISER, T. Ability of dominant follicles (DF) to respond to exogenous $\mathrm{GnRH}$ administration is dependent on their stage of development. Journal of Animal Science, v.71, suppl.1, p.219, 1993.

SOUZA, A.H.; CUNHA, A.P.; CARAVIELLO, D.Z. et al. Profiles of circulating estradiol-17 $\beta$ after different estrogen treatments in lactating dairy cows. Animal Reproduction Science, v.2, n.4, p.224-232, 2005. 
Eficiência do cipionato de estradiol e do benzoato de estradiol em protocolos de indução da ovulação sobre a dinâmica ovariana e taxa de concepção de fêmeas nelore

STEWART, R.E.; SPICER, L.J.; HAMILTON, inseminadas em diferentes momentos

T.D. et al. Effects of insulin-like growth factor I and insulin on proliferation and on basal and luteinizing hormone-induced steroidogenesis of bovine thecal cells: involvement of glucose and receptors for insulin-like growth factor I and luteinizing hormone. Journal of Animal Science, v.73, n.12, p.3719-3731, 1995.

TORRES-JÚNIOR, J.; MELO, W.; ELIAS, et al. Considerações técnicas e econômicas sobre reprodução assistida em gado de corte. Revista Brasileira de Reprodução Animal, v.33, n.1, p.53-58, 2009.

VASCONCELOS, J.L.M.; SARTORI, R.; OLIVEIRA, H.N. et al. Reduction in size of the ovulatory follicle reduces subsequent luteal size and pregnancy rate. Theriogenology, v.56, n.2, p.307-314, 2001.

VIANA, J. H. M.; FERREIRA, A. M.; SÁ, W. F.; CAMARGO, L. S. A. Características morfológicas e funcionais do corpo lúteo durante o ciclo estral em vacas da raça Gir. Arquivo

Brasileiro de Medicina Veterinária e Zootecnia, v.51, n.3, p.251-256, 1999.

VYNCKIER, L. et al. Plasma estradiol-17 beta concentrations in the cow during induced estrus and after injection of estradiol-17 beta benzoate and estradiol-17 beta cypionate--a preliminary study. Journal of Veterinary Pharmacology and Therapeutics, v.13, n.1, p.36-42, 1990. 